\title{
Genetic diversity and population structure of long-tailed macaque (Macaca fascicularis) populations in Peninsular Malaysia
}

\begin{abstract}
Background: The genetic diversity and structure of long-tailed macaques (Macaca fascicularis) in Peninsular Malaysia, a widely used non-human primate species in biomedical research, have not been thoroughly characterized. Methods: Thirteen sites of wild populations of long-tailed macaques representing six states were sampled and analyzed with 18 STR markers. Results: The Sunggala and Penang Island populations showed the highest genetic diversity estimates, while the Jerejak Island population was the most genetically discrete due to isolation from the mainland shelf. Concordant with pairwise Fst estimates, STRUCTURE analyses of the seven PCA-correlated clusters revealed low to moderate differentiation among the sampling sites. No association between geographic and genetic distances exists, suggesting that the study sites, including island study sites, are genetically if not geographically contiguous. Conclusions: The status of the genetic structure and composition of long-tailed macaque populations require further scrutiny to develop this species as an important animal model in biomedical research.
\end{abstract}

Keyword: Crab-eating macaques; Microsatellites; Non-human primates; Population genetics; Short tandem repeats 